\title{
Momentary and integrative response strategies in causal judgment
}

\author{
DARRELL J. COLLINS and DAVID R. SHANKS \\ University College London, London, England
}

\begin{abstract}
Associative models of causal learning predict recency effects: Judgments at the end of a trial series should be strongly biased by recently presented information. Prior research, however, presents a contrasting picture of human performance. López, Shanks, Almaraz, and Fernández (1998) observed recency, whereas Dennis and Ahn (2001) found the opposite, primacy. Here we replicate both of these effects and provide an explanation for this paradox. Four experiments show that the effect of trial order on judgments is a function of judgment frequency, where incremental judgments lead to recency while single final judgments abolish recency and lead instead to integration of information across trials (i.e., primacy). These results challenge almost all existing accounts of causal judgment. We propose a modified associative account in which participants can base their causal judgments either on current associative strength (momentary strategy) or on the cumulative change in associative strength since the previous judgment (integrative strategy).
\end{abstract}

The ability to accurately evaluate causal strength is a central component of adaptive behavior in human and nonhuman animals. We perform an action because it causes a favorable outcome or prevents an unfavorable one; likewise, many decisions, such as those in medicine and economics, rely on making accurate causal judgments (e.g., determining an illness from a set of symptoms or forecasting future economic performance on the basis of current indicators). It is therefore important to delineate the underlying mechanisms that we employ to make these judgments and to determine the sorts of factors that influence them.

Many experimental studies of causal judgment have centered on the $2 \times 2$ task in which participants are presented with information about a single potential cause and effect that are either present or absent from trial to trial, giving four possible types of information. The normative evaluation of the degree of association between the two events is calculated using the $\Delta P$ computation:

$$
\Delta P=P(E / C)-P(E / \sim C)
$$

where $\Delta P$ is the difference between the probabilities of the effect on cause-present versus cause-absent trials. Some research has attempted to explain human performance in causal judgment tasks by stating that this formula or a mechanism derived from it is directly used by humans to detect causal strength (Allan \& Jenkins, 1980;

The research described here was supported by a grant from the United Kingdom Biotechnology and Biological Sciences Research Council. The work is part of the program of the ESRC Centre for Economic Learning and Social Evolution, University College London. Correspondence should be addressed to D. Collins, Department of Psychology, University College London, Gower St., London WC1E 6BT, England (e-mail: d.collins@ucl.ac.uk).
Anderson \& Sheu, 1995; Cheng, 1997; Shaklee \& Tucker, 1980). However, resting on the assumption that the cause and effect in a judgment task are analogous to the conditioned stimulus (CS) and unconditioned stimulus (US) in a Pavlovian conditioning paradigm, a growing body of research originating with Dickinson, Shanks, and Evenden (1984) argues instead that humans develop associative connections between the representations of the cause (or CS) and the outcome (or US). Causal judgments are derived from the strength of these connections. This research led to the application of associative learning models to human causal learning (see Shanks, Holyoak, \& Medin, 1996, for many examples), of which the RescorlaWagner (Rescorla \& Wagner, 1972) model has been the most commonly cited.

The Rescorla-Wagner model states that on each successive presentation of information about a cue-outcome relationship, the strength of association between cue and outcome will be incremented or decremented by a magnitude derived from an error-correcting algorithm formally equivalent to the well-known delta rule. In a typical Pavlovian conditioning task, where a CS is always followed by the US, learning will reach asymptote at a negatively accelerating rate determined by the values of the learning rate parameters. Likewise, in a $2 \times 2$ causal learning task, the model predicts an increase in associative strength following presentation of cause-effect trials and a decrease following cause-no effect trials. According to the model, trials in which the cause is absent will not immediately affect associative strength, but will decrease the amount of associative strength subsequently available to that cause, thus lowering the maximum level of associative strength that can accrue. The level of associative strength obtained following a trial series will closely approximate the normative value defined by $\Delta P$. 
This trial-by-trial updating of associative strength distinguishes the Rescorla-Wagner and other associative models from computational models such as Power PC (Cheng, 1997) in that it is anticipated by the latter that judgments of causality are based on an integration of information across a focal set of trials. Therefore, one clear way of testing the validity of the Rescorla-Wagner model and comparing it with statistical models is to examine the effect of presenting information to participants in different trial sequences.

Perhaps the best illustration of trial-order effects can be seen in simple $2 \times 2$ tasks where the question has been whether information presented early or late in a trial series has the most influence on responses at the end of that series. Because it assumes trial-by-trial updating of associative strength, the Rescorla-Wagner model predicts that the most recent and frequent trial types will have the greatest effect on judgments (i.e., a recency effect). However, research in this domain has not provided clear support for the associative interpretation. On the one hand, López, Shanks, Almaraz, and Fernández (1998) found that responses approximated those predicted by the Rescorla-Wagner model. In a series of experiments, participants were exposed to cue-outcome contingencies where there was an overall null relationship (i.e., $\Delta P=0$ ) but the trial order indicated either a positive then negative contingency or vice versa. Participant evaluations of the cue-outcome contingency were more sensitive to the information presented most recently. Additionally, on each trial following presentation of the cue, participants were asked to predict whether the outcome would occur or not. Comparing the proportion of occasions that participants predicted an outcome on the cue-present trials with that on the cue-absent trials provided an indirect measure of participants' momentary contingency knowledge. This knowledge also tracked the associative strengths predicted by the Rescorla-Wagner model in that participants' predictions incrementally reached an asymptote defined by the cue-outcome contingency.

In contrast, Dennis and Ahn (2001; see also Yates \& Curley, 1986) reported a strong primacy effect that is at odds with the associative interpretation. Participants were required to evaluate the relationship between people ingesting a plant and exhibiting a reaction. Dennis and Ahn constructed trial orders in which the causal relationship between plant and reaction type was highly positive $(\Delta P=.80)$ for the first 20 trials and highly negative $(\Delta P=$ $-.80)$ in the final 20, or vice versa. Following exposure to each set of trials, participants were required to make a judgment about the relationship between plant and reaction. Dennis and Ahn reported that judgments were significantly higher in the positive-negative condition than in the negative-positive condition; that is, they observed a primacy effect. Subsequent experiments indicated that this effect was not due to a decrement in attention across trials or an insufficient number of learning trials.

Since Dennis and Ahn's (2001) results are problematic for all current interpretations of causal learning and especially for associative models, the present program of research begins by replicating their method to determine the robustness of the effects obtained. We then go on to consider the paradox that some conditions lead to primacy and others lead to recency. We demonstrate that the critical determinant of whether or not participants show a recency effect is the frequency with which they make causal ratings. This surprising finding implies that people can adopt different strategies, which we term momentary and integrative, in making causal judgments.

Participants in these experiments were exposed to different trial series in which the cause-outcome relationship between radiation and the development of mutations in butterflies was null overall (i.e., $\Delta P=0$ ) but with a trial order that suggested either a positive and then negative relationship, or vice versa. The only major departures from Dennis and Ahn's (2001, Experiment 1) method were an increase from 40 to 80 trials in each series (although Dennis \& Ahn did use an 80-trial series in a subsequent experiment) and a requirement that participants predict the outcome on a trial-by-trial basis.

\section{GENERAL METHOD}

\section{Participants and Apparatus}

A total of 100 students from University College London volunteered to take part $(12,12,40$, and 36 participants in Experiments 1-4, respectively). They ranged in age from 18 to 50 years with a median age of 23. All participants were tested individually and were paid for their contribution. The task was presented on a Dell OptiPlex GX1 computer connected to a color monitor, with the experimental program written in Visual Basic 5.0.

\section{Procedure}

In all experiments, each participant was exposed to a number of trial series representing the different contingency conditions illustrated in Table 1 (there were four conditions in Experiment 1, and two

Table 1

Event Frequencies in Experiments 1-4

\begin{tabular}{|c|c|c|c|c|}
\hline \multirow[b]{2}{*}{ Condition } & \multicolumn{2}{|c|}{ Block 1} & \multicolumn{2}{|c|}{ Block 2} \\
\hline & Mutation & No Mutation & Mutation & No Mutation \\
\hline \multicolumn{5}{|c|}{ Experiment 1} \\
\hline \multicolumn{5}{|l|}{.75} \\
\hline Radiation & 17 & 3 & 17 & 3 \\
\hline No radiation & 2 & 18 & 2 & 18 \\
\hline-.75 & & & & \\
\hline Radiation & 2 & 18 & 2 & 18 \\
\hline No radiation & 17 & 3 & 17 & 3 \\
\hline \multicolumn{5}{|l|}{$.25 /-.25$} \\
\hline Radiation & 10 & 10 & 5 & 15 \\
\hline No radiation & 5 & 15 & 10 & 10 \\
\hline \multicolumn{5}{|l|}{$-.25 / .25$} \\
\hline Radiation & 5 & 15 & 10 & 10 \\
\hline No radiation & 10 & 10 & 5 & 15 \\
\hline \multicolumn{5}{|c|}{ Experiments 2-4 } \\
\hline $.80 /-.80$ & & & & \\
\hline Radiation & 18 & 2 & 2 & 18 \\
\hline No radiation & 2 & 18 & 18 & 2 \\
\hline$-.80 / .80$ & & & & \\
\hline Radiation & 2 & 18 & 18 & 2 \\
\hline No radiation & 18 & 2 & 2 & 18 \\
\hline
\end{tabular}


in each of the other experiments). Thus, condition is a within-subjects manipulation in all experiments. In Experiment 1, participants made causal judgments after every 10 trials of each series (incremental judgment). In Experiment 2, participants made judgments only after all 80 trials of a trial series (final judgment). In Experiments 3 and 4, participants were systematically allocated to either incremental- or final-judgment conditions as a between-subjects manipulation. In a further between-subjects manipulation, which is not part of our statistical analyses, the trial series were always presented in one of two (three in Experiment 4) preprogrammed, random sequences within the boundaries set by the frequencies shown in Table 1 . These sequences were used equally frequently within each experiment.

After the participants had entered their age and sex, they were presented with the instructions given in the Appendix before completing a brief practice session. Participants were shown laboratory records for each combination of radiation and butterfly species. Following pictorial information about whether the butterfly had been treated with radiation, participants were asked to indicate, using mouse-activate d buttons, either "yes, the mutation is going to occur," or "no, the mutation is not going to occur." They were then informed whether the mutation had occurred. A color image of either a mutated or nonmutated butterfly was shown.

To enable participants to make their causal judgments, a horizontal rating scale was presented on the screen, and the participants were instructed to estimate the degree to which the radiation causes mutations. The scale had endpoints of -100 (radiation prevents mutation) to +100 (radiation causes mutation). Participants made a judgment by moving a slider with the mouse. When the participants were satisfied with their response, they could continue to the next set of records or next trial series.

\section{EXPERIMENT 1}

\section{Method}

Participants in Experiment 1 were exposed to four trial series; these comprised two with a constant $\Delta P(.75,-.75)$ and two with a $\Delta P$ that changed halfway through the 80 -trial series $(.25 /-.25$, $-.25 / .25)$ counterbalanced in a Latin square. Table 1 illustrates the event frequencies from the first (Block 1) and second (Block 2) half of each trial series. Within each condition, trials were presented in one of two possible sequences. In a departure from Dennis and
Ahn's procedure, where only a single rating was taken at the end of each series, participants made causal judgments every 10 trials.

\section{Results and Discussion}

In all of the analyses presented here, a significance level of .05 was employed unless otherwise stated. Figure 1 displays participants' final judgments for each condition. A one-way analysis of variance (ANOVA) revealed a main effect of condition $[F(3,44)=17.02]$. Planned comparisons indicated significant differences between the $.75(M=73.0, S D=7.2)$ and $-.75(M=-59.9, S D=$ $15.4)$ conditions $[t(11)=6.49]$ and between the $.25 /-.25$ $(M=-23.8, S D=15.6)$ and $-.25 / .25(M=28.0, S D=$ $16.5)$ conditions $[t(11)=3.02]$.

Although the order in which information was presented influenced final judgments, it was not in the direction reported by Dennis and Ahn (2001) or Yates and Curley (1986). Rather, consistent with the RescorlaWagner model, judgments were most strongly influenced by information presented in the most recent trials.

\section{EXPERIMENT 2}

The results from the first experiment are in agreement with the predictions of the Rescorla-Wagner model, but the question remains as to why Dennis and Ahn's (2001) results were so different. It seems that the answer to this may lie in differences in method. In Dennis and Ahn's experiments, participants were required to make a judgment only after they had seen all the trials in a series. However, in Experiment 1, participants made judgments after every 10 trials of the series. On the surface this might seem to be a superficial difference, but recent evidence suggests that it may be significant. Catena, Maldonado, and Cándido (1998) demonstrated that accuracy in causal judgments was a function of judgment frequency: Participants were more

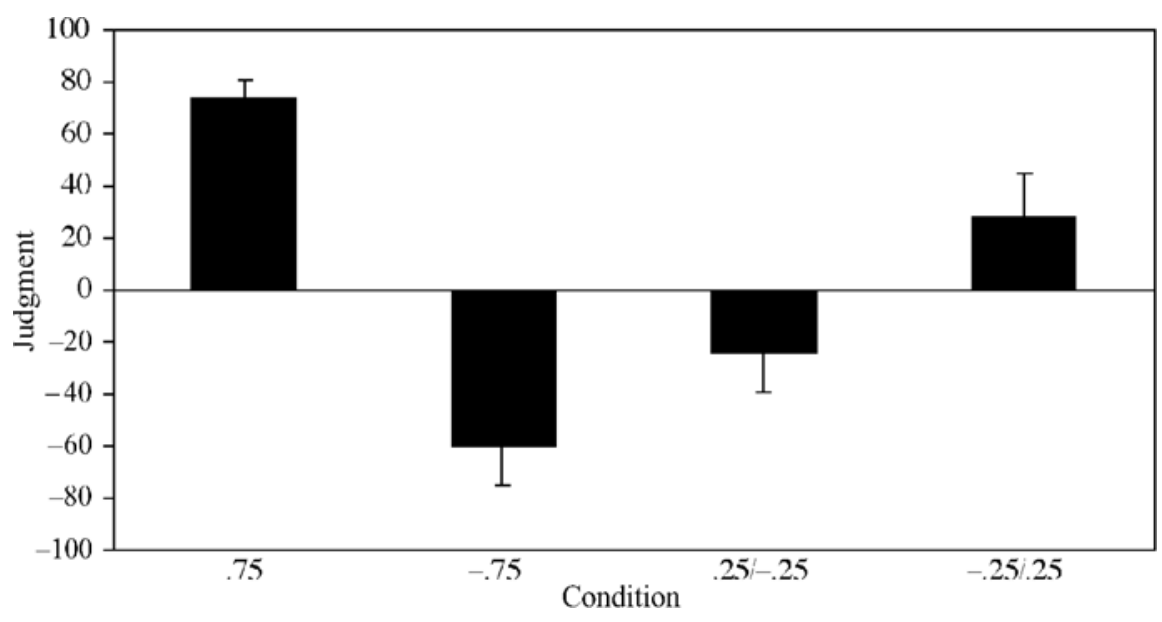

Figure 1. Mean $( \pm S E)$ final judgments of causal strength in a condition with a constant positive $\Delta P(.75)$, a constant negative $\Delta P(-.75)$, in a condition where $\Delta P$ is initially positive and then negative $(.25 /-.25)$, and in a condition where $\Delta P$ is initially negative and then positive $(-.25 / .25)$ in $\mathbf{E x}$ periment 1. 
accurate with longer interjudgment intervals. Final judgments from participants who made judgments over more closely spaced intervals (in this case one or two trials) were sensitive to the information provided on the trial immediately preceding the judgment, indicating that the recency effect might be influenced by the frequency of judgment.

To test the prediction that judgment frequency is responsible for the difference between Dennis and Ahn's (2001) results and those of the present Experiment 1, in Experiment 2 we required the participants to make a judgment only at the conclusion of each 80-trial series.

\section{Method}

Unlike participants in Experiment 1, participants were not required to make causal judgments after every 10 trials. This judgment was requested only at the end of each trial series, using the same scale and instructions as in Experiment 1. In this experiment, participants received two changing conditions, $.80 /-.80$ or $-.80 / .80$, as illustrated in Table 1 . The positive and negative contingencies were therefore more extreme than in the critical conditions of Experiment 1. The present values are the same as those used by Dennis and Ahn (2001).

\section{Results and Discussion}

The mean terminal judgment was $33.2(S D=30.3)$ in the $.80 /-.80$ condition and $6.5(S D=27.6)$ in the $-.80 / .80$ condition. A $t$ test revealed a significant difference between trial-order conditions $[t(11)=2.49]$, indicative of primacy. Contrary to the Rescorla-Wagner model's predictions, it seems in this experiment that participants integrated information from the entire trial series with a larger weighting given to initial information.

A comparison of the proportion of times participants predicted the mutation on radiation versus no-radiation trials allows an indirect measure of their $\Delta P$ estimates to be obtained. In this case, associative models such as that of Rescorla and Wagner (1972) assume that the proportion of predicted outcomes represents a measure of associative strength of the cue compared with the background. Thus, it is possible to track the participants' implicit estimations of the strength of the cue and compare this measure to their direct estimations of the cause-effect relationship. The upper panel of Figure 2 displays the results of this analysis and seems to demonstrate dissociation between participants' implicit evaluation of the cause-effect relationship and their final ratings of that relationship. ${ }^{1}$ Predictions of the outcome follow a pattern of acquisition and sensitivity to changes in the nature of the cause-effect relationship that would be anticipated by associative models of causal learning (i.e., recency). ${ }^{2}$ Thus, when participants are asked explicitly to evaluate causal strength, they do not seem constrained to base their judgments entirely on what their current perception of that relationship is, but instead may integrate information across a number of trials.

\section{EXPERIMENT 3}

It appears from Experiments 1 and 2 that the judgment made at the conclusion of a trial series is to some extent a function of the frequency at which judgments have been made through that series. The results of the previous two experiments suggest that when asked to make frequent judgments, participants use the most recent information they have received (momentary strategy), whereas when an end-of-series judgment is required, they tend to adopt a more global (integrative) judgment strategy. This is akin to the idea of using a moving window of past trials to make a judgment, with the size of the window being demarcated by judgment frequency. In both cases, however, it appears that the participants were sensitive to momentary causal strength, as illustrated by accurate tracking of the cause-effect contingency in their trial-by-trial predictions. In Experiment 3, the judgment conditions of the first two experiments were combined to replicate and better illustrate this judgment frequency effect.

\section{Method}

Participants were assigned to the incremental- or final-judgment conditions as a between-subjects factor. Each participant was exposed to the two contingency conditions presented in Table 1 .

\section{Results and Discussion}

Figure 3 illustrates the mean terminal judgments by condition. A 2 (frequency: incremental, final) $\times 2$ (trial order: .80/-.80, $-.80 / .80)$ mixed ANOVA revealed a significant interaction between trial order and judgment frequency $[F(1,38)=34.70]$. Planned comparisons on terminal judgments revealed that trial order had an impact only in the incremental group $[t(15)=-6.31]$, where strong recency was observed $(.80 /-.80: M=-23.3, S D=$ $39.3 ;-.80 / .80: M=58.4, S D=34.2)$. Although there was numerical primacy in the final-judgment condition, this result failed to reach a conventionallevel of significance $[t(23)=1.20, p=.24]$. The upper panel of Figure 4 illustrates the judgments that participants in the incremental condition made after every 10 trials. It demonstrates that when asked to make an explicit judgment of causal strength, these participants were highly sensitive to recent changes in the status of the cause-effect relationship.

There was a close correspondence between the explicit incremental judgments and the predictions derived from a comparison of the proportion of times the participants predicted a mutation on radiation versus no-radiation trials. The middle panel of Figure 2 illustrates that regardless of the judgment demand placed on the participants, the predictions of the outcome of radiation and nonradiation trials sensitively tracked the changing nature of the event-outcome relationship. The combination of evidence from final and incremental judgments, and from participants' predictions, provides a clear illustration of the frequency-of-judgment effect.

Importantly, this analysis also allows us to rule out an alternative explanation of our results. It is conceivable that participants in the final- and incremental-judgment conditions were interpreting the task differently. Instructions that request incremental judgments might imply instability in the cause-outcome relationship, prime participants to expect a change, and encourage re- 


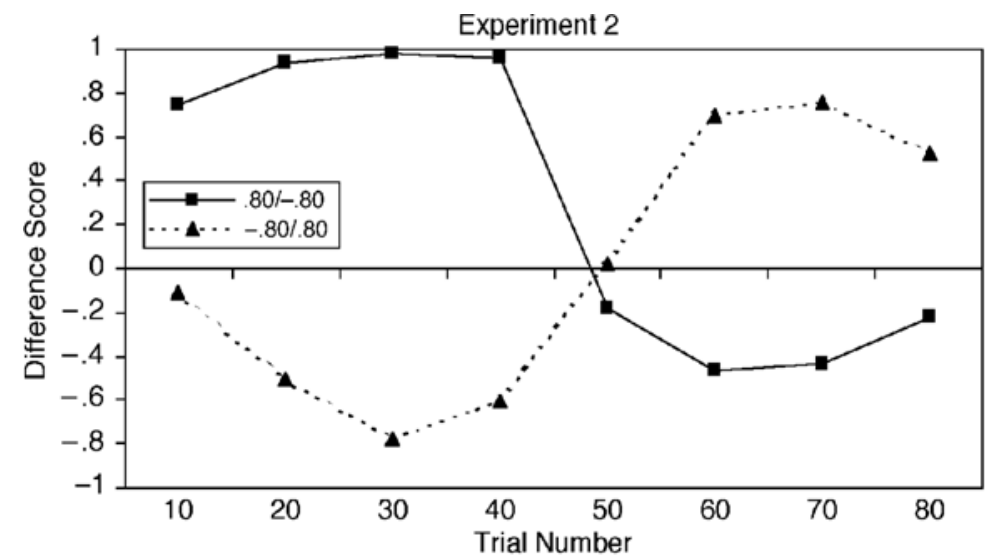

Experiment 3

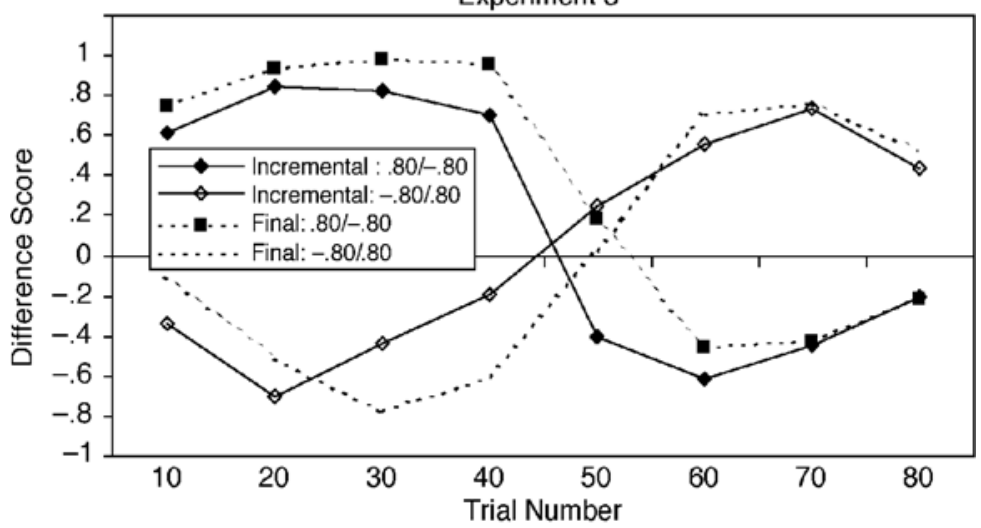

Experiment 4

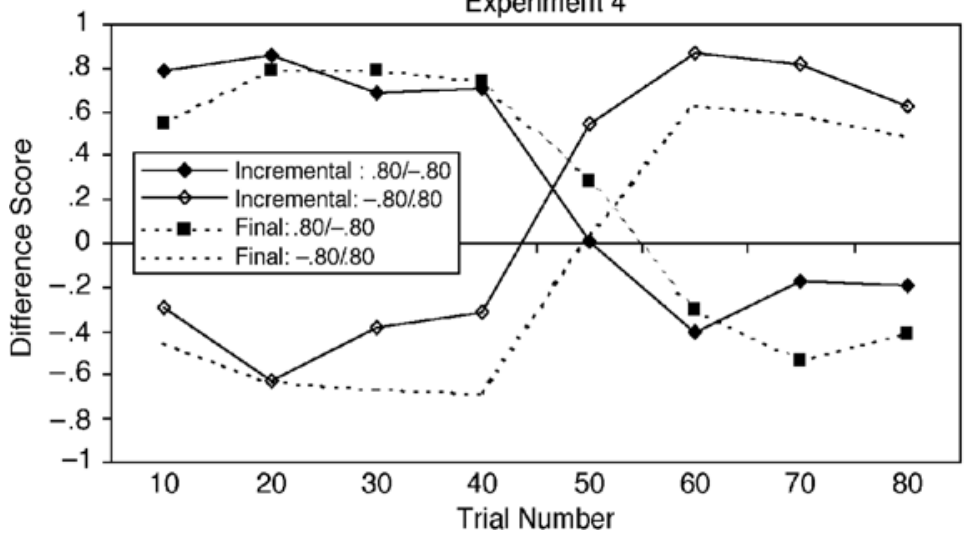

Figure 2. Mean estimates of associative strength derived from prediction data and collapsed over 10-trial blocks for Experiments 2-4. These data were derived by comparing the proportion of times the outcome was predicted on cause-present trials minus the equivalent proportion on cause-absent trials.

cency. In contrast, instructions that ask only for a single judgment at the conclusion of the trial series might induce participants to consider the task as one in which the causal relationship is stable across the series, making them less sensitive to later information. However, the results illustrated in Figure 2 would seem to counter this possibility since participants in both conditions (final, incremental) seemed equally sensitive to the momentby-moment status of the cause regardless of judgment demand. Moreover, if the instructions given to the two groups tended to induce different beliefs about stability, this should be evident only in a between-subjects comparison. In Experiment 4, however, we demonstrated within-subjects exactly the same qualitative pattern ob- 


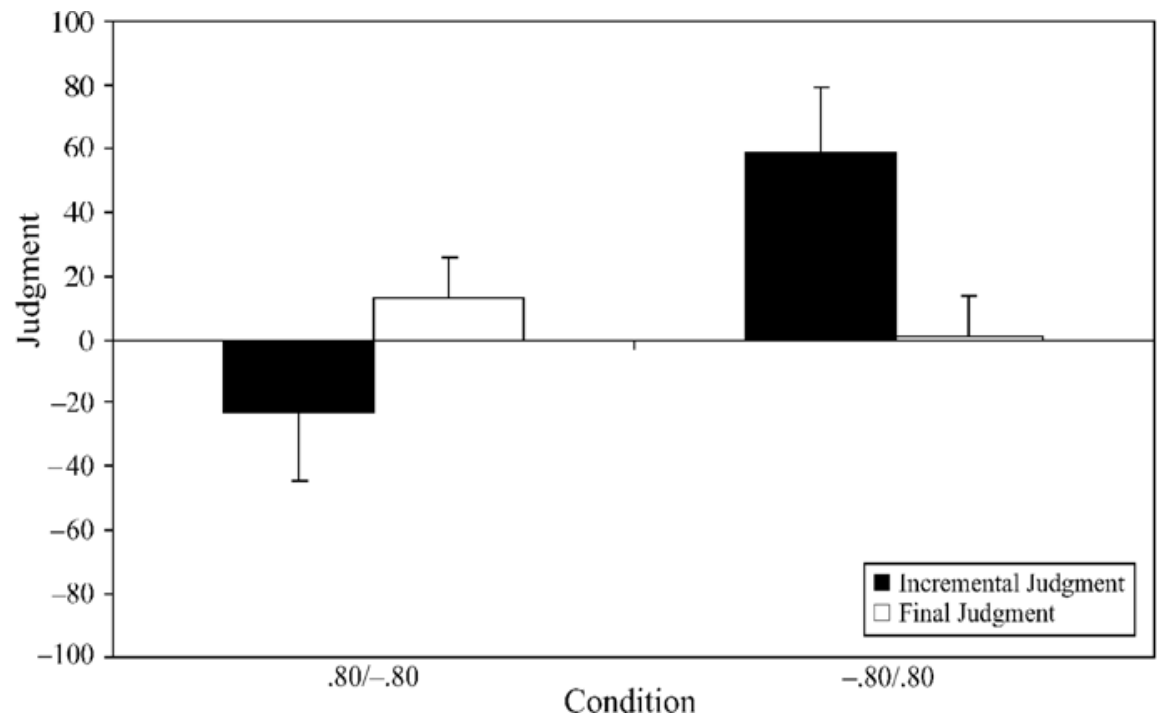

Figure 3. Mean $( \pm S E)$ final judgments of causal strength for the $.80 /-.80$ and $-.80 / .80$ conditions in the incremental- and final-judgment groups in Experiment 3.

served in the final- and incremental-judgment conditions of the present experiment.

Catena et al. (1998) suggested that the interaction between trial order and judgment frequency is evidence for a difference in the nature of information processing when participants have to make regular judgments as opposed to a single final judgment. We conjecture instead that there is a single mechanism of information accumulation but that the frequency-of-judgment effect depends on the participant's selection of one of two possible judgment strategies. In our account, constant across all judgment conditions is an associative learning mechanism responsible for updating the causal status of the cue. This mechanism is apparent from the outcome predictions made on a trial-by-trial basis (Figure 2), which simply reflect the current associative strength of the cue, and we also assume that participants can base their causal judgments on this current associative streng th if they choose. Alternatively, explicit judgments may be based on an integration strategy where participants keep track of the cumulative change in associative strength since their previous judgment and anchor the current judgment to the last one they made (Catena et al., 1998, also suggested an anchoring process). This will lead to a response varying between what appears to be a summary of all information in a final-judgment condition to greater degrees of recency as the frequency of judgment converges with the iterative updating of associative strength.

The idea that participants know not only the current associative strength of a cue but also the changes in associative strength that it has accumulated since the last judgment is a radical modification to associative accounts of causal learning. Typically, there is an assumption of "path independence" in associative theory whereby the route by which a cue got its current strength is unknown; all that is represented is its actual current strength. However, findings in the animal conditioning literature have prompted some to question the path-independence assumption and to propose instead that some aspects of the associative history of a cue are retained (Miller \& Matzel, 1987). Such a proposal is consistent with our finding that judgments made under final-judgment conditions do not show recency.

\section{EXPERIMENT 4}

Our account proposes that participants may adopt one of two strategies when making a causal rating, but we have not yet demonstrated that individual participants can be induced to switch between these strategies at the time of test. To do this, it must be shown that participants, particularly in a final-judgment condition, can use momentary information if required to do so in making causal judgments. In Experiment 4, participants were once again exposed to 80 trials in either an incremental- or final-judgment condition. The critical case is the final-judgment condition. We anticipated that participants in this condition would as before tend to be integrative in their final causal judgments. At the conclusion of each trial series, after making their final judgment, the experimenter presented an extra case and explicitly asked participants to evaluate the causal status of the cue with respect to that case. This causal question is therefore about a specific case rather than about a general causal connection, and we assumed that participants would tend to relate it to the most recent exemplars they had seen. We reasoned that if participants could intentionally adopt a momentary strategy, then the same participants who were integrative in their final judgments should now adopt a mo- 

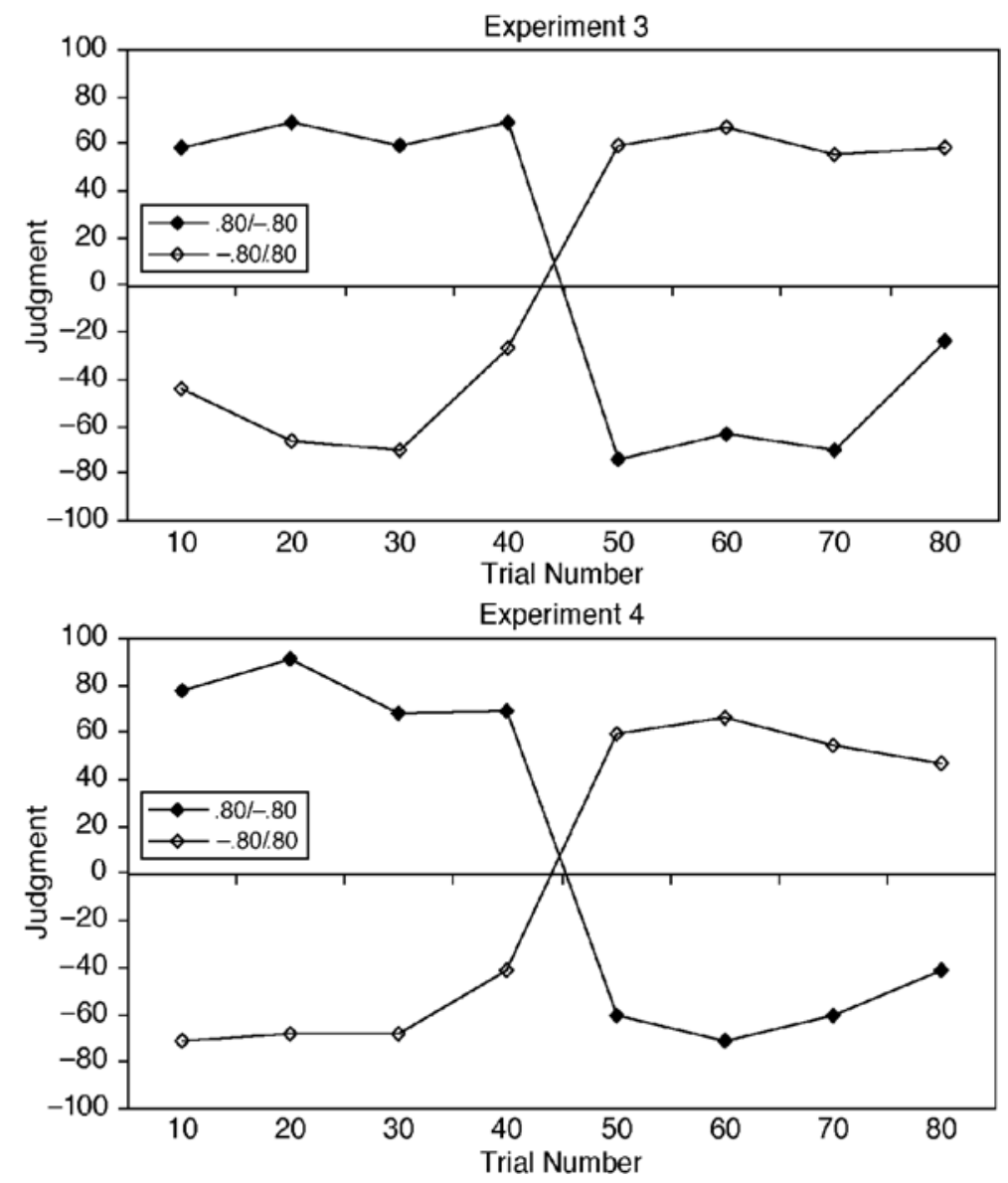

Figure 4. Mean causality judgments from each 10-trial block for the incremental groups in Experiments 3 and 4.

mentary strategy for this additional case and should show a recency effect for this judgment regardless of previous judgment frequency.

\section{Method}

Experiment 4 was identical to Experiment 3 except for the inclusion of an additional test trial at the end of each series of 80 trials. This test consisted of instructing the participant to consider another case, Number 81 . They were informed that this butterfly had been treated with radiation and were asked the following question: "Imagine that Case Number 81 is a butterfly that has received the radiation treatment. Using the scale below, please estimate the degree to which you think the radiation will cause or prevent mutation in this butterfly." Participants were provided with the same scale that had been used for judgments. No additional information was given before the experiment began that the new test trial would be included. In this experiment each condition was instantiated by three rather than two specif ic random trial sequences, which were used equally frequently within each group.

\section{Results and Discussion}

Judgments after 80 trials and on Trial 81, shown in Figure 5, were analyzed separately. A 2 (judgment frequency: incremental, final) $\times 2$ (trial order: .80/-.80, $-.80 / .80)$ ANOVA performed on the Trial 80 judgments revealed a significant interaction between the two factors $[F(1,34)=$
19.94], where a difference in judgments between the two trial-order conditions was apparent only in the incremental group $(.80 /-.80: M=-30.6, S D=43.0 ;-.80 / .80$ : $M=51.8, S D=30.1)[t(17)=5.46]$. This result is consistent with the findings from the previous experiment indicating that participants seem to integrate all the information presented since their last judgment. With regard to the Trial 81 prediction, we were concerned that participants might respond differently in their second trial series than in their first as a result of explicitly anticipating the Trial 81 question. To exclude this possibility we conducted an initial ANOVA with factors of judgment frequency, trial order, and trial series (first, second). There was no main effect or interaction involving trial series, implying that any such anticipation did not influence judgments. Condition order was then removed from an otherwise identical ANOVA, which revealed only a main effect of trial order $[F(1,34)=12.95]$. Regardless of the frequency of judgment required before that point, participant ratings of the causal status of the cue at Trial 81 were always based on the most recent information.

This result demonstrates that participants who seemed to be integrating information across the entire trial series were able to accurately evaluate the momentary causal ef- 

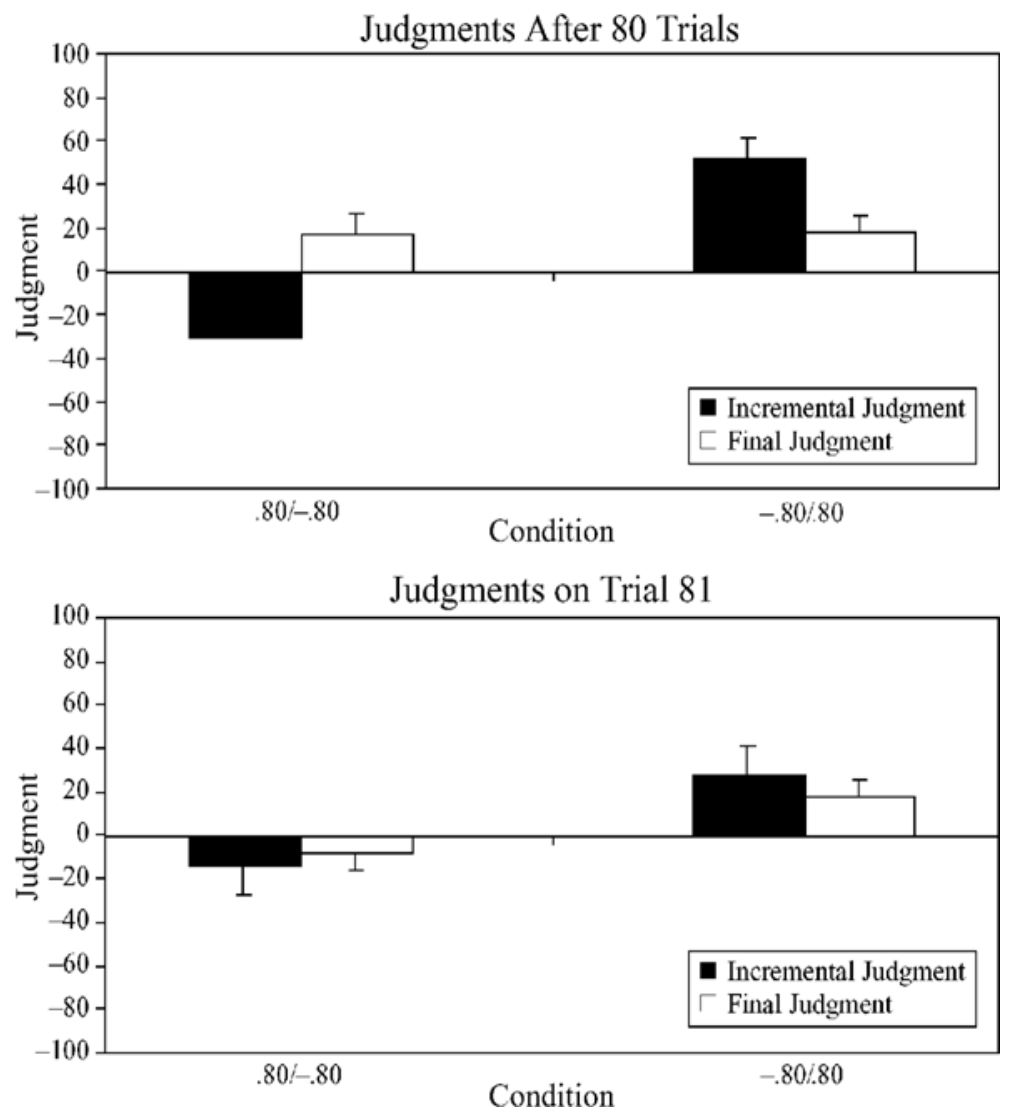

Figure 5. Mean $( \pm S E)$ judgments of causal strength for the $.80 /-.80$ and $-.80 / .80$ conditions in the incremental- and final-judgment groups following Trial 80 and on Trial 81 in Experiment 4.

ficacy of the cue using the most recent information when required to do so. This argues strongly that any differences in judgments between judgment frequency conditions are the result of flexible judgment strategies at test and not differences in information processing during acquisition (Catena et al., 1998).

Comparison of participants' predictions on causepresent and cause-absent trials, illustrated in the bottom panel of Figure 2, once again shows that they closely tracked the actual cause-outcome contingency, as anticipated by the Rescorla-Wagner model. In addition, Figure 4 shows that participants' incremental causality judgments reflected this tracking of the cause-outcome contingency, suggesting again the existence of a single mechanism underlying both processes.

\section{GENERAL DISCUSSION}

The results from the experiments reported here demonstrate that trial-order effects are perhaps not as simple a way of determining the underlying causal learning mechanism as earlier thought. Experiments 1, 3, and 4 demonstrate that participants will give a response that suggests a recency effect when probed for that response relatively frequently. When this judgment is demanded only at the end of a trial series, however, participants tend to integrate the information presented throughout the trial series (Experiments 2-4), although this processing was subject to a slight primacy bias. ${ }^{3}$ Thus, it is likely that the difference in results between this and Dennis and Ahn's (2001) study is due to the frequency of judgment. In both judgment conditions, the trial-by-trial predictions indicated that participants maintain accurate knowledge of the current associative strength of the cue and that this knowledge is sensitive to changes in the cause-outcome relationship. Additionally, Experiment 4 demonstrated that participants are able to draw directly on this knowledge when required to consider a single, specific, case.

Our results bear resemblance to a pattern of findings described by Hastie and Park (1986) in the context of studies of social cognition. They suggested that if a judgment task is "memory-based" and demands a search of memory for relevant evidence (as in the case of the final-judgment condition), previous trials will influence the judgment depending on their availability in a manner similar to that proposed by Kahneman and Tversky (1973). Judgments will therefore tend to show primacy 
if the earlier information from the trial series is more available in memory, and this in turn would be anticipated (see Dennis \& Ahn, 2001) if participants form an initial belief about the causal relationship and if a confirmation bias influences the selection of later information for storage in memory. In contrast, other judgment situations are "online" (as in the case of the incrementaljudgment condition) in the sense that the judgment is continually updated as each piece of evidence is observed. Hastie and Park proposed that under these conditions, participants adopt an "anchor-and-adjust" strategy whereby each judgment is anchored to the previous judgment and adjusted according to the currently presented evidence. Although Hastie and Park did not offer a specific account of the judgment processes engaged in memory-based and online tasks, their review of literature in social cognition suggested that this task difference was important. Plainly, our results fit quite nicely into this framework. Below, we attempt to develop a more detailed model that elaborates the critical processes.

Another cognitive model, the belief-adjustment model (Hogarth \& Einhorn, 1992), proposes a mechanism by which participants can update their estimations of causal strength in a trial-by-trial manner as the basis for either an incremental or final judgment. Hogarth and Einhorn described an anchoring-and-adjustment process in which participants adjust their judgments on the basis of the weighted impact of successive pieces of information (see also Catena et al., 1998). In an incremental condition, the belief-adjustment model generally predicts recency, assuming that each judgment is anchored to the previous one and combined with recently observed evidence. In a final-judgment condition, the same process applies, but in this case averaging of the observed evidence combined with anchoring on the initial evidence conspires to produce primacy. Again, our results are broadly consistent with this framework. Where the present findings go beyond the scope of the belief-adjustment model, and also beyond the scope of Hastie and Park's (1986) classification, is in demonstrating both integrative and momentary judgments simultaneously in the same individual.

The results presented cannot be straightforwardly explained by a statistical model of causal judgment like that of Cheng (1997). In terms of final judgments, this model could explain the frequency-of-judgment effect by assuming that participants employ a moving window that samples only recent trials. Perhaps the size of the moving window is defined by the judgment interval. This would explain the judgment data from the incrementaland final-judgment groups. However, as noted elsewhere (López et al., 1998), the model cannot describe the trialby-trial predictions that participants made, although Cheng has acknowledged that acquisition falls outside the intended scope of her model. The negatively accelerated shapes of the functions shown in Figure 2 cannot be explained by appeal to $P(E / C)$ and $P(E / \sim C)$, the key determinants of judgments according to theories such as Cheng's, since these probabilities are constant across trials within each phase of the experiment. Instead of ac- counting for the data in terms of a single underlying information-accumulation process combined with different judgment strategies (as we have proposed), a more radical idea would be to assume that participants learn both associative relationships and records of the relevant conditional probabilities. Hence Cheng's theory, with varying sizes of moving window, could apply to the causal judgments, whereas an associative mechanism would explain trial-by-trial predictions.

Associative models such as that of Rescorla and Wagner (1972) do not provide a full explanation of the data either. Because of the way associative strength is updated after each trial, the Rescorla-Wagner model always predicts recency effects. As a result, regardless of the frequency of the probe question, participants should always base their judgments on the current associative strength of the cause. The experiments reported here clearly indicate that this is not the case. At one level (implicit predictions), it appears that participants are basing their judgments directly on what would be expected to be the current level of associative strength. However, when explicit judgments are requested, there seems to be an additional process occurring that the Rescorla-Wagner model is unable to describe. This model also has difficulty explaining other empirical results (e.g., Perales \& Shanks, in press), which has led researchers to consider alternative associative accounts such as those of Pearce (1987) and van Hamme and Wasserman (1994). But since these models also employ error-driven learning mechanisms, they would be similarly troubled by our results.

We have suggested that a simple addition to the associative mechanism described by the Rescorla-Wagner model could explain this difference. Consider a situation in which participants encode the cumulative change in associative strength of the cue from when they were last probed for it. When a new judgment is demanded, this change is critical, and the current judgment is a function of this change combined with an anchoring effect of the last judgment. In tasks where frequent causal judgments are requested, this will lead to recency, since estimates will be made close to the rate at which associative strength is updated. In contrast, when the frequency of judgment is low, judgments of causality may not bear much resemblance to the current status of the cue if the cause-outcome relationship has changed.

On this account, participants effectively have two strategies at their disposal for making causal judgments. The integrative strategy, based on cumulative change in associative strength, may be replaced by a momentary one in which the current associative strength forms the basis for judgments. In Experiment 4, the same participants who tended to be integrative in making their judgments in the final-judgment condition tended to be momentary in making judgments about a specific new case.

\section{REFERENCES}

Allan, L. G., \& Jenkins, H. M. (1980). The judgment of contingency and the nature of the response alternatives. Canadian Journal of Psychology, 34, 1-11. 
Anderson, J. R. \& Sheu, C.-F. (1995). Causal inferences as perceptual judgments. Memory \& Cognition, 23, 510-524.

Catena, A., Maldonado, A., \& CÁndido, A. (1998). The effect of the frequency of judgment and the type of trials on covariation learning. Journal of Experimental Psychology: Human Perception \& Performance, 24, 481-495.

Cheng, P. W. (1997). From covariation to causation: A causal power theory. Psychological Review, 104, 367-405.

Dennis, M. J., \& AHn, W.-K. (2001). Primacy in causal strength judgments: The effect of initial evidence for generative versus inhibitory relationships. Memory \& Cognition, 29, 152-164.

Dickinson, A., Shanks, D. R., \& Evenden, J. L. (1984). Judgment of act-outcome contingency: The role of selective attribution. Quarterly Journal of Experimental Psychology, 36A, 29-50.

HAstie, R. \& PARK, B. (1986). The relationship between memory and judgement depends on whether the judgment task is memory-based or on-line. Psychological Review, 93, 258-268.

Hogarth, H. J., \& Einhorn, R. M. (1992). Order effects in belief updating: The belief-adjustment model. Cognitive Psychology, 24, 1 55 .

Kahneman, D., \& Tversky, A. (1973). Availability: A heuristic for judging frequency and probability. Cognitive Psychology, 5, 207-232.

López, F. J., Shanks, D. R., Almaraz, J., \& Fernández, P. (1998). Effects of trial order on contingency judgments: A comparison of associative and probabilistic contrast accounts. Journal of Experimental Psychology: Learning, Memory, \& Cognition, 24, 672-694.

Miller, R. R, \& Matzel, L. D. (1987). Memory for associative history of a conditioned stimulus. Learning \& Motivation, 18, 118-130.

Pearce, J. M. (1987). A model for stimulus generalization in Pavlovian conditioning. Psychological Review, 94, 61-73.

Perales, J. C., \& Shanks, D. R. (in press). Normative and descriptive accounts of the influence of power and contingency on causal judgment. Quarterly Journal of Experimental Psychology.

Rescorla, R. A., \& Wagner, A. R. (1972). A theory of Pavlovian conditioning: Variations in the effectiveness of reinforcement and nonreinforcement. In A. H. Black \& W. F. Prokasy (Eds.), Classical conditioning II: Current research and theory (pp. 64-99). New York: Appleton-Century-Crofts.

ShaKleE, H., \& TuCKer, D. (1980). A rule analysis of judgments of covariation between events. Memory \& Cognition, 8, 459-467.

Shanks, D. R, Holyoak, K. J., \& Medin, D. L. (Eds.). (1996). The psychology of learning and motivation: Vol.34. Causal learning. San Diego: Academic Press.

van Hamme, L. J., \& Wasserman, E. A. (1994). Cue competition in causality judgments: The role of nonpresentation of compound stimulus elements. Learning \& Motivation, 25, 127-151.

Yates, J. F., \& Curley, S. P. (1986). Contingency judgment: Primacy effects and attention decrement. Acta Psychologica, 62, 293-302.

\section{NOTES}

1. A similar analysis was conducted for Experiment 1. However, because the contingencies in the changing conditions varied across a smaller range than in this experiment, the differences in the implicit estimations were not as marked as they are here.

2. Figures 2 and 4 reveal a slight tendency for difference scores and incremental judgments to converge in the final block of trials in the present experiment; a similar "pincer" effect emerges in Experiment 3 and, to a lesser extent, in Experiment 4. This tendency is due to the fact that, by chance, the two trial sequences we constructed tended to have values of $\Delta P$ in the final block that were closer to zero than the preceding blocks (specifically, in Experiments 2 and 3, the contingency in each successive 10 -trial block in the $-.80 / .80$ condition was either -1.0 , $-.8,-.8,-.63,1.0, .87, .80, .66$, or $-1.0,-.8,-.5,-.3,1, .75, .8$, .58 , depending on which specific trial sequence the participant was exposed to; the contingencies in the $.80 /-.80$ condition were the exact converse of these). Thus, the pincer effect in the acquisition curves was due to random fluctuations in experienced contingency and not to par- ticipants' estimates drifting away from asymptote. In Experiment 4 we added a third preprogrammed trial sequence to partially ameliorate this fluctuation; Figures 2 and 4 confirm that the pincer effect was reduced if not entirely eliminated in that experiment.

3. In Experiment 2, a strong primacy effect was observed, but the effect was somewhat weaker in Experiments 3 and 4. When data from the three experiments were combined, the mean final judgments were 18.8 $(S D=28.9)$ in the $.80 /-.80$ condition and $6.1(S D=31.8)$ in the $-.80 / .80$ condition, indicative of primacy. A 3 (experiment) $\times 2$ (trial order) ANOVA confirmed a main effect of trial order $[F(1,51)=4.61]$ and no experiment $\times$ trial-order interaction.

\section{APPENDIX}

\section{Experimental Instructions}

Imagine you are working in a laboratory and you want to find out whether certain types of radiation cause or prevent genetic mutations in butterflies' DNA. During this task you will see laboratory records from four studies. In each study, you will see information about administering one type of radiation to one species of insect.

In one study, Gonepteryx formosana were irradiated with $\mathrm{U} 256$ nuclear radiation, in another Ixias pyrene were irradiated with P290 [in another Ixias reinwardti were irradiated with W119, and in a fourth Catopsilia scylla were irradiated with Z210].* In each study, some butterflies received nuclear radiation and others did not. After a period of time the butterflies' DNA was examined for genetic mutations.

Of course, DNA mutations sometimes occur spontaneously in insects not exposed to nuclear radiation. What you must decide is whether the radiation increases or decreases the likelihood of mutations.

There are 80 butterflies in each study. Each record tells you whether the butterfly was exposed to the relevant nuclear radiation or not. You will then be asked to predict whether or not the butterflies' DNA will show a genetic mutation. When you have made your prediction you will be told whether a mutation was found or not. Use this feedback to try to find out whether the radiation really causes or prevents mutations. Although initially you will have to guess, by the end you will be an expert!

During each study you will be asked from time to time to estimate [At the end of each study you will be asked to estimate] ${ }^{\dagger}$ the degree to which the radiation causes or prevents mutations. Using a scale, you will indicate a number between -100 and +100 , where -100 indicates that the radiation prevents mutations, 0 indicates that it neither causes nor prevents mutations, and 100 indicates that the radiation causes mutations. Intermediate numbers indicate intermediate levels of cause or prevention. You can use any number on the slider; the numbers provided are only a guide.

You can now try some practice trials. Once they are over you may start the experiment, look at the instructions again, or have more practice.

*Instructions reflected the number of conditions in the experiment.

${ }^{\dagger}$ Participants in the final-judgment condition were therefore aware that they would make one final judgment only, whereas those in the incremental condition were aware that they would make a number of judgments through each trial series.

(Manuscript received September 19, 2001; revision accepted for publication May 31,2002.) 